\section{Probing the phase transition of aqueous solutions of linear low molecular weight poly $(N$-isopropylacrylamide) by dielectric spectroscopy}

\author{
Marieke Füllbrandt, ${ }^{a}$ Regine von Klitzing ${ }^{b}$ and Andreas Schönhals ${ }^{* a}$
}

Received 7th August 2012, Accepted 25th September 2012

DOI: $10.1039 / \mathrm{c} 2 \mathrm{sm} 26826 \mathrm{~d}$

\begin{abstract}
Aqueous solutions of linear poly( $N$-isopropylacrylamide) (pNIPAM) with different polymer concentrations are investigated using dielectric spectroscopy in a frequency range of $10^{-1} \mathrm{~Hz}$ to $10^{6} \mathrm{~Hz}$ at temperatures from $15{ }^{\circ} \mathrm{C}$ to $50{ }^{\circ} \mathrm{C}$. The phase transition of pNIPAM is monitored by both the temperature $(T)$ and the frequency $(f)$ dependence of the conductivity spectra $\sigma^{*}(f, T)$. First, the $T$-dependence of the DC conductivity $\sigma_{\mathrm{DC}}^{\prime}$ is investigated and the phase transition ("coil-to-globule" transition) at the lower critical solution temperature (LCST) of PNIPAM is deduced by a change in the $T$-dependence of $\sigma_{\mathrm{DC}}^{\prime}$. The observed hysteresis between heating and cooling runs is discussed in detail in dependence on both the polymer concentration and the rate. Second, for the first time a pronounced $f$-dependence of the real part of conductivity $\sigma^{\prime}$ is observed at temperatures above the LCST whereas at temperatures below the LCST the conductivity spectra are more or less similar to that of water (frequency independent). This $f$-dependence of $\sigma^{\prime}$ is assigned to the formation of a kind of soft particle ("globular structure") at the LCST which is more or less impermeable to water and ions therefore giving rise to Maxwell-Wagner-Sillars (MWS) polarization effects (blocking of charges at the soft particle). The dependence on the concentration is studied in detail.
\end{abstract}

\section{Introduction}

"Smart" polymeric materials have received an increasing attention because these systems respond in a reversible and more important in a controlled way with a property change to a weak external stimulus, such as temperature, light, electric and magnetic fields, as well as ionic strength. ${ }^{1,2} \operatorname{Poly}(N$-isopropyl acrylamide) (pNIPAM) is one of the most widely studied thermosensitive materials. It has gained a growing interest in the last decades due to its unique thermal properties. Potential applications in biotechnology and medicine, including drug release and diagnostics, chemical separation, catalysis, surface modification, etc. have been suggested. ${ }^{3-5}$ In aqueous media pNIPAM shows a lower critical solution temperature (LCST) at around $32{ }^{\circ} \mathrm{C}^{6}$ which was first reported by Heskins and Guillet in $1968 .{ }^{7}$ At temperatures below its LCST the polymer is hydrophilic and swollen in water, whereas at temperatures above the LCST a phase separation occurs. This process is reversible and for linear pNIPAM it is called "coil-to-globule" transition since polymer chains pass from a swollen coil conformation to a globular one. The globular structure of pNIPAM chains is suggested to have different chain densities and to consist of a dense "core" and a

${ }^{a} B A M$ Bundesanstalt für Materialforschung und -prüfung, Unter den Eichen 87,12205 Berlin, Germany.E-mail: andreas.schoenhals@bam.de; Fax: +49-30-81041637; Tel: +49-30-81043384

${ }^{b}$ Stranski-Laboratorium für Physikalische und Theoretische Chemiel Institut für Chemie, Technische Universität Berlin, Strasse des 17. Juni 124, 10623 Berlin, Germany more swollen soft "shell" having also chain loops. ${ }^{8,9}$ Therefore, in a certain sense it can be considered as a nanoscaled soft particle, i.e. a particle with a hard inner core and a soft shell being a more porous and permeable layer. ${ }^{10}$

The transition phenomenon is related to the competition between two molecular effects: the hydrophobic behaviour of the pendent isopropyl groups as well as that of the backbone and the hydrogen bonding between the amide groups and water molecules (solvent). ${ }^{11}$ The "coil-to-globule" (heating) as well as the "globule-to-coil" (cooling) transitions are complex processes and pass different states. ${ }^{12,13}$ The transition is a phenomenon widely studied because it resembles certain processes occurring in biological systems, such as protein folding and native DNA packing, but it is also related to the collapse of a gel network. ${ }^{14-16}$ The temperature-induced phase separation of aqueous pNIPAM solutions provides an appropriate model to study the molecular interactions in biological systems and thermoreversible hydrogels. Although a lot of work was done on the swelling/shrinking properties, the kinetics of the transition during the temperature increase (or decrease) and its relation to the internal structure are far from being understood.

Linear pNIPAM systems have been characterized by various methods including light scattering, ${ }^{13,17}$ infrared spectroscopy, ${ }^{18,19}$ differential scanning calorimetry, ${ }^{20-22}$ fluorescence microscopy, ${ }^{23}$ turbidimetry ${ }^{21,24}$ and NMR. ${ }^{25,26}$ In this paper dielectric spectroscopy is applied for the investigation of aqueous solutions of linear pNIPAM. Dielectric spectroscopy is a well suited technique to study the structure and dynamics of polymers including their 
solutions. It allows investigations of the molecular structure by taking the molecular mobility and/or the conductivity as a probe. ${ }^{27}$

Ono and Shikata ${ }^{28,29}$ applied dielectric spectroscopy at high frequencies $\left(1.6 \times 10^{6} \mathrm{~Hz}\right.$ to $\left.1.6 \times 10^{11} \mathrm{~Hz}\right)$ in order to study the hydration and molecular dynamics of linear pNIPAM in aqueous solutions at temperatures between $6{ }^{\circ} \mathrm{C}$ and $39^{\circ} \mathrm{C}$. The temperature dependence of the (de)hydration behaviour of the monomer NIPAM and the polymer pNIPAM was investigated by analyzing the dynamics of water molecules hydrated to the solute. The relaxation processes of linear pNIPAM and solvent molecules as a function of polymer concentration and type of solvent have been studied by Nakano et al. ${ }^{30}$ Dielectric spectroscopy was applied in a frequency range from $4 \times 10^{4} \mathrm{~Hz}$ to $2 \times 10^{10} \mathrm{~Hz}$ at a temperature of $25^{\circ} \mathrm{C}$. Two relaxation processes could be detected due to the fluctuations of the polymer segments (MHz range) and the solvent molecules (GHz range). Conclusions about the dynamics among polymer segments and solvent molecules were drawn. However, in general only little is known about the molecular (including the segmental) dynamics of pNIPAM, especially in the temperature region of the phase transition. Recently, a new device for measurements of optical and dielectric properties for linear pNIPAM and hydrogels in aqueous solutions has been presented by Gómez-Galván et al. ${ }^{31}$ The light transmittance, the relative permittivity and the dielectric loss tangent as a function of temperature were measured in the lower frequency region $\left(10^{2} \mathrm{~Hz}\right.$ to $\left.10^{6} \mathrm{~Hz}\right)$ in order to analyse the water-polymer interaction.

In this study the temperature $(T)$ and frequency $(f)$ dependence of the complex conductivity $\sigma^{*}(f, T)$ obtained by dielectric spectroscopy of aqueous pNIPAM solutions in a wide range of concentrations from the dilute to the concentrated regime are investigated and discussed. Suited parameters are deduced from the dielectric spectra and correlated with the transition behaviour as well as with the corresponding conformational changes of the pNIPAM chains for the first time. The $T$ - and $f$-dependence of the conductivity give information about the amount of charge carriers (density), the arrangement of these charges as well as their mobility and transfer processes. No extra charge carriers have been added to the pNIPAM solution, only the remaining charges coming from the polymerization process and residual charge carriers in the Milli- $\mathrm{Q}^{\mathrm{TM}}$ water used as the solvent have been considered. The general approach is to use charges as probes to investigate the internal structure of the pNIPAM solution. Here the well-studied linear pNIPAM is used as a model system in order to demonstrate that dielectric spectroscopy is a useful method to investigate these materials. In the future this method will be applied to more complex systems where the information about internal morphology and transition kinetics obtained by well established techniques like light scattering might be limited. Moreover in contrast to other methods a wide concentration range can be covered.

\section{Experimental}

\section{Materials and sample preparation}

Linear poly( $N$-isopropylacrylamide) (pNIPAM) was purchased from Sigma Aldrich. The molecular weight characterizations by Gel Permeation Chromatography (GPC), matrix-assisted laser desorption/ionization (MALDI) and osmometry resulted in a molecular weight of 2500-3000 $\mathrm{g} \mathrm{mol}^{-1}$. GPC gives a number average molecular weight $M_{\mathrm{n}}$ of $2300 \mathrm{~g} \mathrm{~mol}^{-1}$ with a PDI of 1.8 .

Aqueous pNIPAM solutions with different concentrations were prepared by dissolving a defined amount of polymer $(c=$ $1 \mathrm{mg} \mathrm{ml}^{-1}$ to $150 \mathrm{mg} \mathrm{ml}^{-1}$ ) in Milli-Q ${ }^{\mathrm{TM}}$ water (Millipore, USA).

\section{Dielectric spectroscopy}

The complex dielectric function $\varepsilon^{*}$ is derived by measuring the complex impedance $Z^{*}(\omega)$ of the sample:

$$
\varepsilon^{*}(\omega)=\varepsilon^{\prime}(\omega)-i \varepsilon^{\prime \prime}(\omega)=\frac{1}{i \omega Z^{*}(\omega) C_{0}}
$$

where $\varepsilon^{\prime}$ is the real part, $\varepsilon^{\prime \prime}$ the loss part, $\omega$ the angular frequency $(f=2 \pi \omega), i=(-1)^{1 / 2}$ and $C_{0}$ the capacitance of the empty sample capacitor. $Z^{*}(\omega)$ is measured using Fourier Correlation Analysis. ${ }^{27}$ Measurements were carried out in a frequency range from $10^{-1} \mathrm{~Hz}$ to $10^{6} \mathrm{~Hz}$ and between $15^{\circ} \mathrm{C}$ and $50^{\circ} \mathrm{C}$ with a high resolution ALPHA analyser (Novocontrol, Hundsangen, Germany). The temperature is controlled by a Quatro Novocontrol cryo system with a temperature stability of $0.1 \mathrm{~K}$. The polymer solutions are filled in a liquid sample cell supplied by Novocontrol (BDS 1309) with a diameter of $11 \mathrm{~mm}$. The cell is made up of two gold plated electrodes with a Teflon cylinder as the spacer in between. The electrode spacing of $5.5 \mathrm{~mm}$ is relatively wide in order to shift the effect of electrode polarization to lower frequencies.

\section{Results and discussion}

In the considered temperature range $\left(15{ }^{\circ} \mathrm{C}\right.$ to $\left.50{ }^{\circ} \mathrm{C}\right)$ the molecular fluctuations of water and the segmental mobility of pNIPAM take place at much shorter time scales (higher frequencies). ${ }^{29,30}$ They cannot be observed in the selected frequency range as in characteristic relaxation processes. However, conductivity related phenomena like the drift motion of charge carriers (DC-conductivity) and/or interfacial polarization effects such as Maxwell-Wagner-Sillars (MWS) polarization can be detected. The dielectric data can be presented in different representations, such as complex dielectric permittivity, electrical modulus or complex conductivity $\sigma^{*}(\omega)$, containing identical information but emphasising different aspects of polarization and charge transport processes. ${ }^{27}$ Here, the focus is on the mobility of charges, therefore the data are expressed for complex conductivity which is given by:

$$
\sigma^{*}(\omega)=\sigma^{\prime}(\omega)+i \sigma^{\prime \prime}(\omega)=i \omega \varepsilon_{0} \varepsilon^{*}(\omega)
$$

where $\sigma^{\prime}$ and $\sigma^{\prime \prime}$ are the corresponding real and loss parts, respectively ( $\varepsilon_{0}$ is the permittivity of free space). For details see ref. 27.

With methods like dynamic light scattering only dilute systems can be investigated. This is not the case for dielectric spectroscopy where the concentration of the solution can be varied in a broad range from the dilute to the concentrated regime. Moreover, state of the art dielectric equipment allows a fast data sampling. Therefore both concentration and kinetic effects can be addressed. 
As an example Fig. 1 compares the frequency $f$ and temperature $T$ dependence of the real part $\sigma^{\prime}$ of complex conductivity for water (Milli- $\mathrm{Q}^{\mathrm{TM}}$ ) and an aqueous solution of pNIPAM. For a conductive system the real part $\sigma^{\prime}$ of the complex conductivity should be independent of frequency. This value of $\sigma^{\prime}$ is the DC conductivity of the system. Exactly this is observed for pure water in a wide frequency range (see Fig. 1a). The conductivity is due to the drift motion of charge carriers which are present in the system mainly due to impurities in the ppm concentration range which could not be removed during purification. In addition protonic effects (hopping of protons between water molecules) can contribute to the mechanism of conduction for water. ${ }^{32}$

For frequencies lower than $1 \mathrm{~Hz}$ a slight decrease of $\sigma^{\prime}$ with decreasing frequency is seen in the dielectric spectra of water which is due to electrode polarisation. ${ }^{33}$ At a fixed frequency $\sigma^{\prime}$ increases continuously with increasing temperature as expected (a detailed discussion will be given in the next section).

In contrast to pure water the conductivity spectrum of the pNIPAM solution (see Fig. 1b) shows a pronounced frequency as well as temperature dependence. These dependencies are related to both the drift motion of charge carriers (DC conductivity) and MWS polarization effects due to the formation of the globular structure (soft particle) at the lower critical solution temperature (LCST). Both dependencies will be discussed in detail in the following.
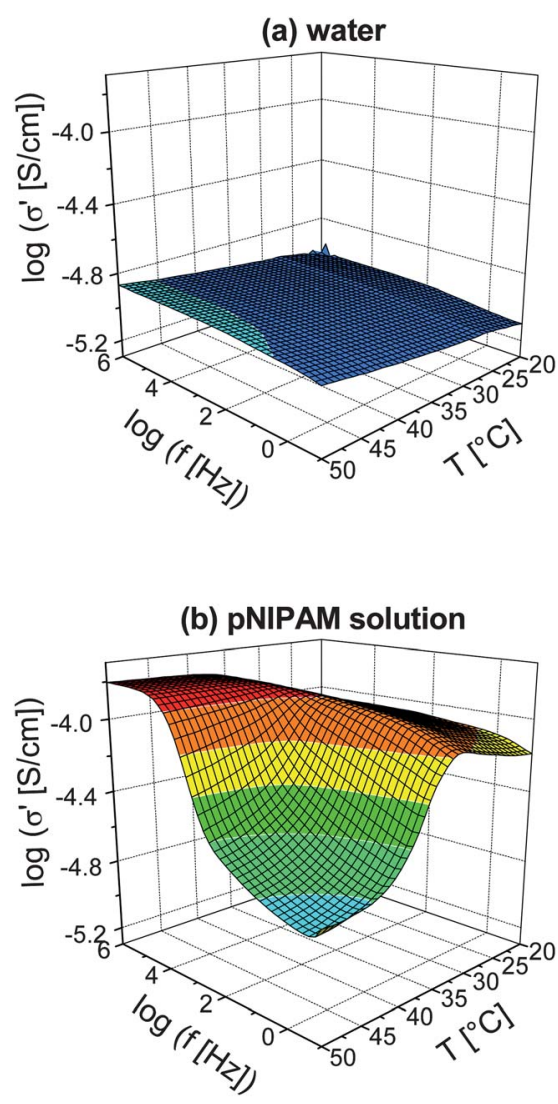

Fig. 1 Real part of the complex conductivity $\sigma^{\prime}$ versus frequency and temperature in a $3 \mathrm{D}$ representation for (a) Milli- $\mathrm{Q}^{\mathrm{TM}}$ water and (b) an aqueous solution of pNIPAM $\left(c=25 \mathrm{mg} \mathrm{ml}^{-1}\right)$.

\section{Temperature dependence of the DC conductivity $\sigma_{\mathrm{DC}}^{\prime}$}

At high frequencies the conductivity $\sigma^{\prime}$ of the pNIPAM solution is independent of the frequency similar to water. This plateau region corresponds to the DC conductivity of the system $\left(\sigma_{\mathrm{DC}}^{\prime}\right)$ in the extrapolation $f \rightarrow 0 \mathrm{~Hz}$. For the pNIPAM solutions the mechanism of conduction is also caused by drift motion of charge carriers which are already present in the systems. Because additional impurities (traces of catalysts from synthesis, etc.) are introduced $\sigma_{\mathrm{DC}}^{\prime}$ for the pNIPAM solutions is slightly higher than for water. To skip interfacial polarization effects (which mostly take place in the lower and middle frequency regions) for a first discussion the DC conductivity $\sigma_{\mathrm{DC}}^{\prime}$ of aqueous pNIPAM solutions is examined in its temperature dependence. The samples were heated from $15{ }^{\circ} \mathrm{C}$ to $50{ }^{\circ} \mathrm{C}$ in steps of $1 \mathrm{~K}$ or $2 \mathrm{~K}$ and subsequently cooled down to $15{ }^{\circ} \mathrm{C}$. At each temperature an isothermal frequency scan is carried out. This results in an effective heating/cooling rate of $0.2 \mathrm{~K} \mathrm{~min}^{-1}$. As discussed above for pure water $\sigma_{\mathrm{DC}}^{\prime}$ increases continuously with the temperature since the conductivity is proportional to the mobility of charge carriers which increases with the temperature (see Fig. 2). The absolute value of $\sigma_{\mathrm{DC}}^{\prime}$ at $25^{\circ} \mathrm{C}$ and $45^{\circ} \mathrm{C}$ is around $10 \mu \mathrm{S} \mathrm{cm}$ and $12 \mu \mathrm{S} \mathrm{cm}^{-1}$, respectively. The conductivity for the pNIPAM solution $\left(c=25 \mathrm{mg} \mathrm{ml}^{-1}\right)$ is around ten times higher compared to that of water $\left(100 \mu \mathrm{S} \mathrm{cm}^{-1}\right.$ at $25^{\circ} \mathrm{C}$ and $154 \mu \mathrm{S} \mathrm{cm}^{-1}$ at $45^{\circ} \mathrm{C}$ for the heating scan) due to a larger amount of charges coming from impurities remaining from the polymerization process. In contrast to water, for the pNIPAM solution a change in the temperature dependence of $\sigma_{\mathrm{DC}}^{\prime}$ is observed (see Fig. 2). Two approximately linear regimes in $\sigma_{\mathrm{DC}}^{\prime}(T)$ can be identified, the one at lower temperatures having a steeper slope than the one at higher temperatures. From the intersection of the two linear fits to the corresponding data a phase transition temperature can be deduced. ${ }^{34}$ A similar behaviour was observed for all other pNIPAM concentrations. The molecular origin of the change in the temperature dependence of $\sigma_{\mathrm{DC}}^{\prime}$ for pNIPAM solutions will be discussed in more detail in the course of this contribution.

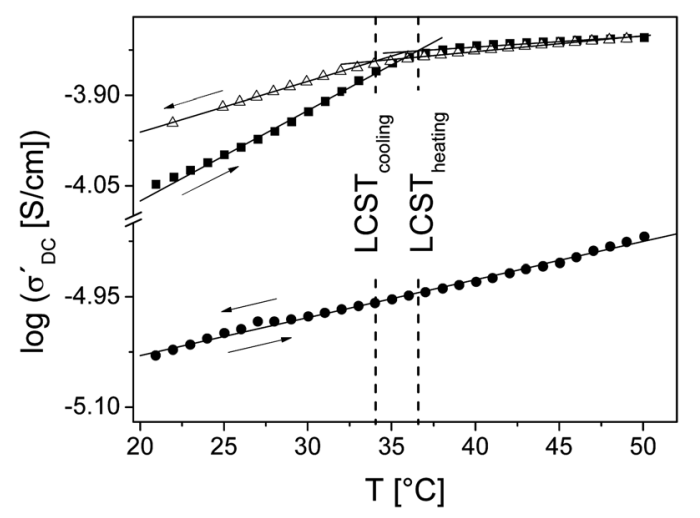

Fig. 2 Temperature dependence of the DC conductivity $\sigma_{\mathrm{DC}}^{\prime}$ for Milli$\mathrm{Q}^{\mathrm{TM}}$ water (filled circles) and a pNIPAM solution with $c_{\mathrm{pNIPAM}}=25 \mathrm{mg}$ $\mathrm{ml}^{-1}$ (upper curves) (filled squares $=$ heating scan, open triangles $=$ cooling scan). Solid lines are linear fits to the data, dashed lines indicate the change in slope (= LCST) in the case of the pNIPAM solution. The arrows in right and left directions mark the heating and cooling runs, respectively. (Please note the break in the $y$-axis.) 
Comparing the LCST values taken from heating and cooling a hysteresis is observed. The "coil-to-globule" (heating) transition is located at a higher temperature than the reverse "globule-tocoil" process (cooling). Similar effects were observed by dynamic light scattering for dilute aqueous solutions of pNIPAM $^{17}$ and by ultrasensitive differential scanning calorimetry for semidilute pNIPAM solutions. ${ }^{22}$

This is attributed to kinetic effects for instance the formation of additional hydrogen bonds in the collapsed state which hinder the refolding/dissolution of the globules during cooling. ${ }^{9,13,16}$ In addition different slopes for heating and cooling are observed below the LCST which is an indication that the formation and the dissolving of globules follow different time scales (see also the discussion on Fig. 5).

For a more quantitative discussion the extent of the hysteresis is expressed by $\Delta \mathrm{LCST}=\mathrm{LCST}_{\text {heating }}-\mathrm{LCST}_{\text {cooling, }}$, and discussed in its dependence on the polymer concentration (see Fig. 3). As a general result the $\Delta$ LCST increases with increasing polymer concentration. This might be due to a structural change of the solution which will lead to the formation of aggregates at higher concentrations. According to the theory of polymer solution one has to distinguish between dilute and semidilute or concentrated states where polymer coils overlap and interact. The change from isolated coils to overlapping ones can be characterized by the overlap concentration $C^{*}$ given by: ${ }^{35}$

$$
C^{*}=\frac{3 M}{4 \pi N_{\mathrm{A}} R_{\mathrm{g}}{ }^{3}}
$$

where $M$ and $R_{\mathrm{g}}$ are the molecular mass and the radius of gyration of the polymer chain, respectively, and $N_{\mathrm{A}}$ is the Avogadro number. According to the literature ${ }^{36}$ for a linear pNIPAM with $M_{\mathrm{w}}=7 \times 10^{3} \mathrm{~g} \mathrm{~mol}^{-1}$ and a hydrodynamic radius $R_{\mathrm{h}}$ of $3 \mathrm{~nm} C^{*}$ is about $30 \mathrm{mg} \mathrm{ml}^{-1}$ at $25^{\circ} \mathrm{C}$ where the radius of gyration is calculated by $R_{\mathrm{h}}=2 / 3 R_{\mathrm{g}}$ according to the Kirkwood-Riseman theory. ${ }^{37}$ Assuming a radius of gyration $R_{\mathrm{g}}$ of $3.9 \mathrm{~nm}$ for the pNIPAM under study with $M_{\mathrm{w}}=3 \times 10^{3} \mathrm{~g}$ $\mathrm{mol}^{-1}$ (based on ref. 36) $C^{*}$ is estimated to be around $20 \mathrm{mg}$ $\mathrm{ml}^{-1}$. This calculated overlapping concentration is in agreement

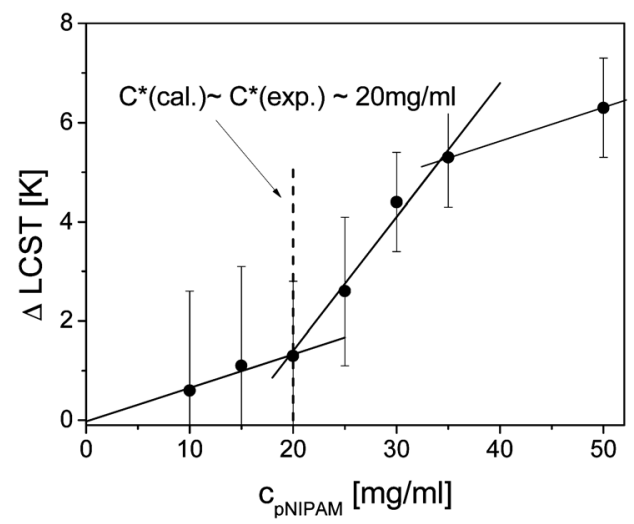

Fig. $3 \Delta \mathrm{LCST}$ (= $\mathrm{LCST}_{\text {heating }}-\mathrm{LCST}_{\text {cooling }}$ ) versus polymer concentration. The solid lines are linear fits to the data. The dashed line indicates the overlap concentration $C^{*}$ at $c_{\mathrm{pNIPAM}}=20 \mathrm{mg} \mathrm{ml}^{-1}$ according to both the calculation described in the text as well as the interception of the linear fits to the experimental data. The bars represent typical errors deduced from different linear regressions for the $\Delta$ LCST determination (number of used data points). The heating/cooling rate is $0.2 \mathrm{~K} \mathrm{~min}^{-1}$. with the pNIPAM concentration of around $20 \mathrm{mg} \mathrm{ml}^{-1}$ where the slope of $\Delta$ LCST versus concentration changes abruptly (see Fig. 3). In the more condensed state different polymer coils interpenetrate which will then probably lead to larger globules for $T>$ LCST. These larger structures will be further stabilized by the formation of hydrogen bonds involving different chains. In terms of the transition temperature it means that with increasing polymer concentration the LCST is shifted to higher temperatures in the heating cycle and lower temperatures in the cooling cycle resulting in a more pronounced hysteresis. The second change in the concentration dependence of the $\triangle \mathrm{LCST}$ at around $40 \mathrm{mg} \mathrm{ml}^{-1}$ might indicate the transition to an even more condensed state.

To study the time dependence of the hysteresis more deeply, measurements were carried out at a fixed frequency of $f=5.85 \times$ $10^{5} \mathrm{~Hz}$, where the heating and cooling rate was varied between $0.1 \mathrm{~K} \mathrm{~min}^{-1}$ and $5 \mathrm{~K} \mathrm{~min}^{-1}$. Results for a pNIPAM solution $(c=$ $25 \mathrm{mg} \mathrm{ml}^{-1}$ ) are presented in Fig. 4. With increasing heating/ cooling rate the extent of the hysteresis becomes more pronounced since the system has less time to relax and adopts its final (equilibrium) conformation at each temperature. That is, the chain contraction and association/aggregation (heating) as well as the dissolution of the collapsed pNIPAM chains (cooling) cannot follow the temperature change.$^{38}$ For the lowest heating/ cooling rate $\left(0.1 \mathrm{~K} \mathrm{~min}^{-1}\right)$ the equilibration time at each temperature seems to be long enough so that there is no hysteresis effect left, i.e. the "coil-to-globule" and "globule-to-coil" transitions are observed at the same temperature. At low rates the $\triangle$ LCST increases strongly with the rate whereas for higher rates a kind of plateau is observed. To analyse the data in more detail $\triangle$ LCST is plotted versus the logarithm of the rate (inset of Fig. 4). The data can be well described by a straight line implying a logarithmic dependence of the hysteresis on the rate with $\Delta$ LCST $[\mathrm{K}]=9.35+7.94 \log \left(\right.$ rate $\left.\left[\mathrm{K} \mathrm{min}^{-1}\right]\right)$ in the considered range. An extrapolation to zero $\Delta$ LCST results in a rate of $0.07 \mathrm{~K}$ $\min ^{-1}$ confirming that the LCST at $0.1 \mathrm{~K} \mathrm{~min}^{-1}\left(36^{\circ} \mathrm{C}\right)$ can be

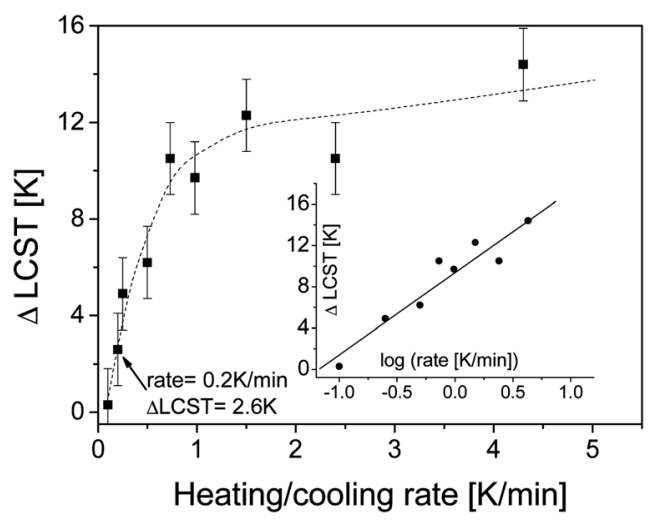

Fig. 4 Dependence of the $\Delta \mathrm{LCST}$ ( $=\mathrm{LCST}_{\text {heating }}-\mathrm{LCST}_{\text {cooling }}$ ) on the heating/cooling rate for an aqueous solution of pNIPAM $(c=25 \mathrm{mg}$ $\mathrm{ml}^{-1}$ ). The dashed line is a guide to the eyes. The bars represent typical errors deduced from different linear regressions for LCST determination (number of used data points). The arrow marks the standard heating/ cooling rate for all other experiments $\left(0.2 \mathrm{~K} \mathrm{~min}^{-1}\right)$ corresponding to a $\Delta \mathrm{LCST}$ of $2.6 \mathrm{~K}$ for $c_{\mathrm{pNIPAM}}=25 \mathrm{mg} \mathrm{m}^{-1}$ (compare Fig. 3). In the inset $\Delta$ LCST is plotted versus the logarithm of the rate. The line is a linear regression to the data. 


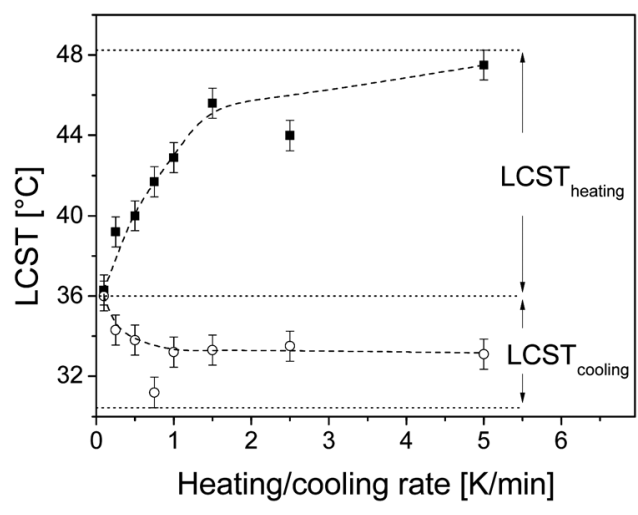

Fig. 5 Dependence of $\mathrm{LCST}_{\text {heating }}$ (filled squares) and LCST $\mathrm{Looling}_{\text {(open }}$ circles) on the heating/cooling rate for an aqueous solution of pNIPAM $\left(c=25 \mathrm{mg} \mathrm{ml}^{-1}\right)$. The dashed lines are guides to the eyes. The dotted lines mark the temperature range for $\mathrm{LCST}_{\text {heating }}$ and $\mathrm{LCST}_{\text {cooling, }}$ respectively. The bars represent typical errors deduced from different linear regressions (the number of data points used for fitting is varied).

taken as the equilibrium transition temperature for the system under investigation.

The transition temperatures for heating ( $\left.\operatorname{LCST}_{\text {heating }}\right)$ and cooling $\left(\mathrm{LCST}_{\text {cooling }}\right.$ ) are plotted versus the rate in Fig. 5 in order to gain a deeper understanding of the hysteresis behaviour. The values for $\mathrm{LCST}_{\text {heating }}$ depend much stronger on the rate than the corresponding values for $\mathrm{LCST}_{\text {cooling }}$ which are located in a narrow temperature range. An increase in $\mathrm{LCST}_{\text {heating }}$ and a decrease in $\mathrm{LCST}_{\text {cooling with increasing rate for an aqueous }}$ pNIPAM solution were also observed by Ding et al. using ultrasensitive differential scanning calorimetry. ${ }^{22,38}$ They also reported that with increasing cooling rate the aggregated chains only partially dissolved during cooling and aggregation can be only removed by a certain incubation time at $T<\mathrm{LCST}^{22}{ }^{22}$ The plateau reached at about $1 \mathrm{~K} \mathrm{~min}^{-1}$ for the $\mathrm{LCST}_{\text {cooling }}$ and the overall weaker dependence of $\mathrm{LCST}_{\text {cooling }}$ on the cooling rate can be explained by an incomplete dissolution of the aggregated chains at cooling rates higher than $1 \mathrm{~K} \mathrm{~min}^{-1}$. In this case the structural conformation of the pNIPAM solution at the starting and end temperature of the measurement $\left(15^{\circ} \mathrm{C}\right)$ is probably not the same.

Furthermore, one can conclude from Fig. 5 that the observed hysteresis is mainly due to the strong increase of $\mathrm{LCST}_{\text {heating }}$ with the heating rate. This observation points out that different time scales are needed for the formation and the dissolving of hydrogen bonds, which is also shown in Fig. 2 by the different $T$-dependence of the pNIPAM solution in the heating and cooling scans. Above the LCST the change of the conductivity with temperature of the pNIPAM solution is similar for heating and cooling whereas below the LCST the behaviour is different and the variation of the conductivity with temperature is weaker for the cooling than for the heating run.

\section{Frequency dependence of the complex conductivity $\sigma^{*}(f)$ (interfacial polarization effects)}

The frequency dependence of the complex conductivity $\sigma^{*}$ of the pNIPAM solutions was investigated at temperatures below and above the phase transition. The conductivity spectra are normalized with respect to the value of the DC conductivity discussed above for the sake of comparison. This means log $\sigma_{\text {norm }}^{\prime}=\log \left[\sigma^{\prime}(f) / \sigma_{\text {DC }}^{\prime}\right]$. Milli-Q ${ }^{\mathrm{TM}}$ water shows only a weak frequency dependence of the real part of complex conductivity $\sigma^{\prime}$. For high frequencies $\sigma^{\prime}$ is constant (DC plateau) where the slight decrease of $\sigma^{\prime}$ with decreasing frequency at lower frequencies is due to electrode polarization (see Fig. 6). For aqueous pNIPAM solutions at temperatures below the LCST the frequency dependence is similar to that of water. But however for temperatures above the LCST a pronounced frequency dependence of $\sigma^{\prime}$ is observed. This is demonstrated in Fig. 6. The fact that the pronounced frequency dependence of $\sigma^{\prime}$ is only observed for temperatures above the LCST leads to the conclusion that it is caused by the structural change of the pNIPAM chains in solution for temperatures $T>\mathrm{LCST}$ and the related change in charge transport processes including its arrangement.

With increasing temperature at the phase transition the pNIPAM chains collapse, associate and adapt a globular conformation, which can be considered as a "soft" particle. The structure of the whole system changes from a more or less homogeneous solution to an inhomogeneous particle suspension. A dielectric signature of inhomogeneous systems is the so-called Maxwell-Wagner-Sillars (MWS) polarization ${ }^{27}$ due to the blocking and separation of charge carriers at phase boundaries. Roughly spoken the separated charges of opposite polarity can be considered as a kind of dipole giving rise to a polarization. The reorientation of this dipole in the alternating field causes a frequency dependence of $\sigma^{*}(f)$ in addition to a simple DC conductivity. For the pNIPAM system considered here charges can be blocked at the interfaces of the formed soft particles for $T>$ LCST at a mesoscopic length scale. The resulting polarization causes a strong decrease in the real part of conductivity $\sigma^{\prime}$ with decreasing frequency. Therefore the corresponding frequency dependence of $\sigma^{\prime}$ is assigned to a MWS polarization due to the presence of soft particles (globules) for temperatures above the LCST. A comparable frequency dependent behaviour of the conductivity was predicted by the Hanai model for colloidal dispersions with spherical particles. ${ }^{39,40}$

However, when investigating conductive materials, e.g. aqueous suspensions, the unwanted parasitic effect of electrode

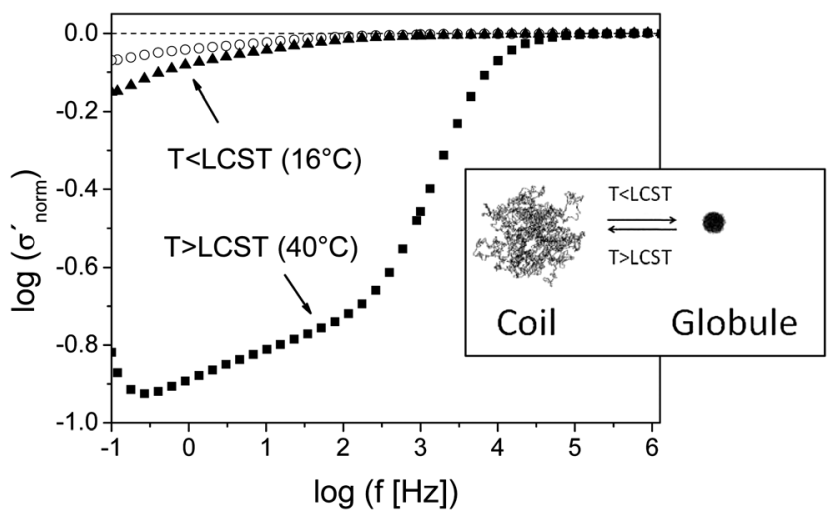

Fig. 6 Frequency dependence of the real part of the normalized conductivity $\sigma_{\text {norm }}^{\prime}$ (curves are normalized to the corresponding value of $\left.\sigma_{\text {DC }}^{\prime}\right)$. Open circles: Milli- $\mathrm{Q}^{\mathrm{TM}}$ water at $16^{\circ} \mathrm{C}$, filled triangles: pNIPAM solution $\left(c=25 \mathrm{mg} \mathrm{ml}^{-1}\right)$ at $16{ }^{\circ} \mathrm{C}$, and filled squares: pNIPAM solution $\left(c=25 \mathrm{mg} \mathrm{ml}^{-1}\right)$ at $40{ }^{\circ} \mathrm{C}$. 
polarization has to be also considered. It is observed on a macroscopic scale at lower frequencies and similar to MWS effects due to the accumulation of charges, in this case at the surface of the electrodes. It causes the formation of electrical double layers at the electrodes at a macroscopic length scale which contributes strongly to the measured dielectric quantities $\left(\sigma^{*}, \varepsilon^{*}\right)$ due to the sample material alone. ${ }^{27}$ The characteristic signatures of electrode polarizations are discussed in detail in ref. 33 for the examples of ionic liquids considering both real and imaginary parts of the complex permittivity and conductivity.

In a related approach in order to elucidate characteristic effects of MWS polarization and to discriminate between electrode and MWS polarization in Fig. 7 the real part of permittivity $\varepsilon^{\prime}$ and the imaginary part of conductivity $\sigma^{\prime \prime}$ are plotted versus frequency for water (a) and a pNIPAM solution with $c=$ $25 \mathrm{mg} \mathrm{ml}^{-1}$ (b). For pure water only electrode polarization is expected. In that case $\varepsilon^{\prime}$ shows a strong increase for frequencies lower than $10^{3} \mathrm{~Hz}$. In parallel in the loss part of the conductivity $\sigma^{\prime \prime}$ a minimum is observed. This can be considered as the onset of electrode polarization (when going from high to low frequencies). ${ }^{33}$ Moreover, the behaviour slightly depends on the temperature (see Fig. 7a). Here, the onset frequency of electrode polarization for water is located at around $1 \mathrm{kHz}$.

Below the LCST the pNIPAM solution shows a similar dielectric behaviour to water characterized by an onset frequency of electrode polarization of $4.5 \mathrm{kHz}$. This onset frequency is slightly higher than that for pure water due to a higher conductivity of the pNIPAM solution (see Fig. 2). For temperatures above the LCST the frequency dependence of $\sigma^{\prime \prime}$ and $\varepsilon^{\prime}$ changes completely, indicating that another process in addition to electrode polarization takes place. For $T=40^{\circ} \mathrm{C}(T>\mathrm{LCST})$ $\sigma^{\prime \prime}$ shows with decreasing frequency a first minimum located at $f=115.6 \mathrm{kHz}$ followed by a maximum situated at $f=3.01 \mathrm{kHz}$. The corresponding real part of the complex permittivity $\varepsilon^{\prime}$ shows a step-like increase also in pronounced difference to $\varepsilon^{\prime}$ for $T<$ LCST. The behaviour of the complex conductivity as well as permittivity is assigned to a MWS polarization with an onset frequency of $115.6 \mathrm{kHz}$ (corresponding to the minimum of $\sigma^{\prime \prime}$ ). a) pure water
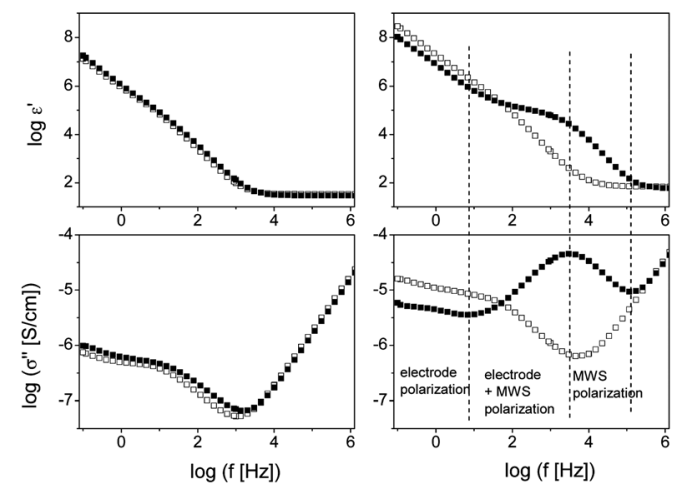

Fig. 7 Real part of the permittivity $\varepsilon^{\prime}$ (top panels) and imaginary part of the conductivity $\sigma^{\prime \prime}$ (bottom panels) versus frequency for water (a) and a solution of linear pNIPAM (b) with $c=25 \mathrm{mg} \mathrm{ml}^{-1}$ at $16{ }^{\circ} \mathrm{C}$ (open squares) and $40^{\circ} \mathrm{C}$ (filled squares). The dashed lines indicate the three $f$-regions for $T=40{ }^{\circ} \mathrm{C}$ where different polarization processes (as indicated) take place.
Table 1 Frequency regions with corresponding polarization effects (plus $(+)$ indicates "yes", minus (-) indicates "no") extracted from Fig. 7 for a pNIPAM solution with $c_{\text {pNIPAM }}=25 \mathrm{mg} \mathrm{ml}^{-1}$

\begin{tabular}{llll}
\hline $\begin{array}{l}\text { Polarization } \\
\text { effect }\end{array}$ & $\begin{array}{l}\text { Low } f \text {-region } \\
(\sim 0.1 \text { to } 7 \mathrm{~Hz})\end{array}$ & $\begin{array}{l}\text { Middle } f \text {-region } \\
(\sim 7 \text { to } 3000 \mathrm{~Hz})\end{array}$ & $\begin{array}{l}\text { High } f \text {-region } \\
(\sim 3 \text { to } 115 \mathrm{kHz})\end{array}$ \\
\hline $\begin{array}{l}\text { Electrode } \\
\text { MWS }\end{array}$ & + & + & - \\
\hline
\end{tabular}

According to the nomenclature given in ref. 33 at the maximum of $\sigma^{\prime \prime}$ the MSW polarization can be considered as fully developed.

The frequency spectra of the pNIPAM solutions at $T>$ LCST can be divided into three regions determined by the minima and maximum of $\sigma^{\prime \prime}$ (see Fig. 7) as listed in Table 1.

In the low frequency regime the absolute value of $\varepsilon^{\prime}$ for temperatures above the LCST is lower than for temperatures below the LCST (see Fig. 7b, upper panel). Due to the blocking of the charge carriers at the formed soft particles the charge carrier density is reduced at the electrodes leading to a less pronounced electrode polarization expressed by a lower value of $\varepsilon^{\prime}$ in comparison to temperatures below the LCST where charge carriers are not blocked.

The reduced charge carrier density at $T>$ LCST is also discussed as the molecular origin of the change in the temperature dependence of DC conductivity at the phase transition. The DC conductivity $\sigma_{\mathrm{DC}}^{\prime}$ can be expressed as a product of the number density $N$ of charge carriers and their mobility $\mu$. Both quantities will be affected by the phase transition. On the one hand, due to blocking of charge carriers at the soft particles their number density is reduced. In addition, also due to the presence of soft particles the remaining charges have a longer mean diffusion way to reach the electrodes leading to a lower effective mobility. Both effects should lead to a decrease of the conductivity above the LCST. On the other hand the conductivity generally increases with the temperature. The changed slope $\operatorname{d} \log \sigma / \mathrm{d} T$ at the phase transition (see Fig. 2) is therefore due to a counterbalance of the increase of the conductivity with the temperature at the one side

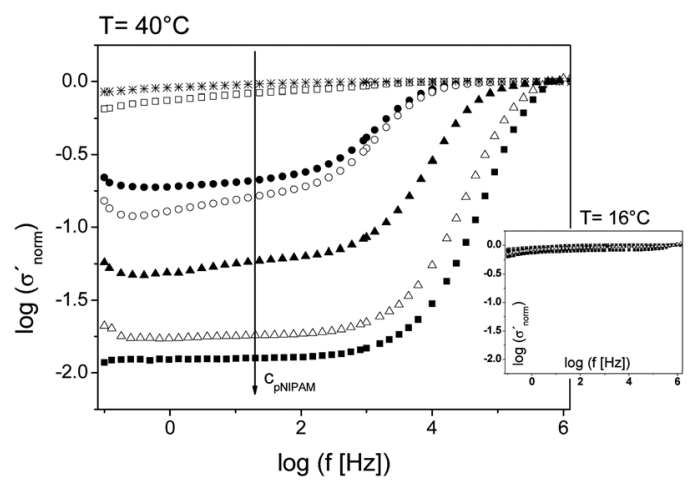

Fig. 8 Frequency dependence of the real part of conductivity $\sigma_{\text {norm }}^{\prime}$ for aqueous solutions of pNIPAM with different concentrations at $40{ }^{\circ} \mathrm{C}$ and $16{ }^{\circ} \mathrm{C}$ (inset). Filled squares: $c=150 \mathrm{mg} \mathrm{ml}^{-1}$, open triangles: $c=$ $100 \mathrm{mg} \mathrm{ml}^{-1}$, filled triangles: $c=50 \mathrm{mg} \mathrm{ml}^{-1}$, open circles: $c=25 \mathrm{mg}$ $\mathrm{ml}^{-1}$, filled circles: $c=10 \mathrm{mg}$, open squares: $c=1 \mathrm{mg} \mathrm{ml}^{-1}$, and stars: $c=$ $0 \mathrm{mg} \mathrm{ml}^{-1}$ (water). 
and the reduced number of charge carriers with a lower effective mobility at the other side.

In Fig. 8 the frequency dependence of $\sigma^{\prime}$ at $T=16^{\circ} \mathrm{C}$ and $T=$ $40{ }^{\circ} \mathrm{C}$ for different polymer concentrations is shown. For temperatures below the LCST the normalized curves for all concentrations collapse more or less onto a single chart showing only weak frequency dependencies due to electrode polarization (see the inset of Fig. 8). At temperatures above the LCST MWS polarization effects are observed which become more pronounced with increasing polymer concentration. For a more quantitative analysis, the step increase in $\sigma^{\prime}$ at $T>$ LCST is analysed with respect to the position of its point of deflection and its height in dependence on the polymer concentration and temperature. The position of the point of deflection $\left(\log f_{\text {defl }}\right)$ was determined by the position of the maximum of the first derivative shown in Fig. 9 (upper panel). Fig. 10a shows the frequency of the point of deflection $\log f_{\text {defl }}$ in dependence on the polymer concentration. Generally speaking, the frequency position is shifted to higher frequencies with increasing amount of polymer. Note that the particle density increases with increasing concentration. This means in turn that the average distance between the particles becomes smaller and particles associate and aggregate. Since the time constant for MWS polarization depends on the distance between the particles ${ }^{41}$ and decreases with decreasing particle distance, a shorter time (i.e. higher frequency) is required for charge blocking at interfaces. This explains the shift of the MWS polarization to higher frequencies with increasing particle concentration. The distance between the point of deflection and the DC conductivity plateau is defined as half of the step height $h / 2$ (see Fig. 9, bottom panel). The dependence of $h / 2$ on the polymer concentration for three temperatures above the LCST is shown in Fig. 10b. As already indicated in Fig. 8, the MWS polarization effect increases with increasing polymer concentration. This can be attributed to two effects. First, with increasing
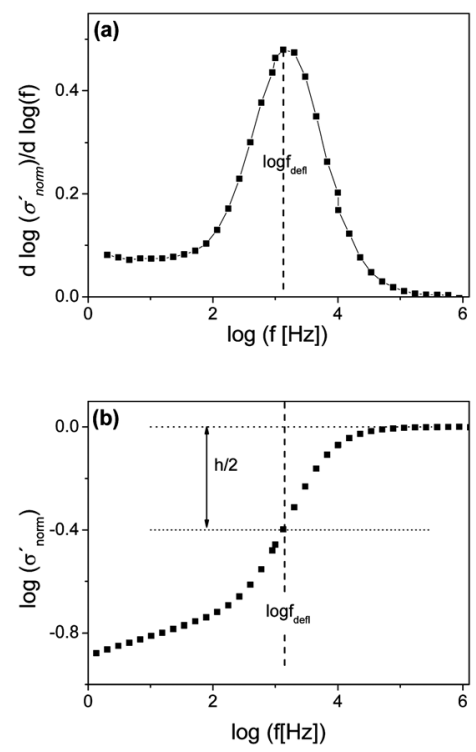

Fig. 9 Quantitative analysis of the frequency dependence of the real part of conductivity $\sigma^{\prime}$. (a) First derivative (d $\log \sigma_{\text {norm }}^{\prime} / \operatorname{dlog} f$ ) for the pNIPAM solution of $25 \mathrm{mg} \mathrm{ml}^{-1}$ where the position of the maximum is taken as the position of the point of deflection $\log f_{\text {defl }}$. (b) Definition of the step height $h / 2$ for the pNIPAM solution of $25 \mathrm{mg} \mathrm{ml}^{-1}$.
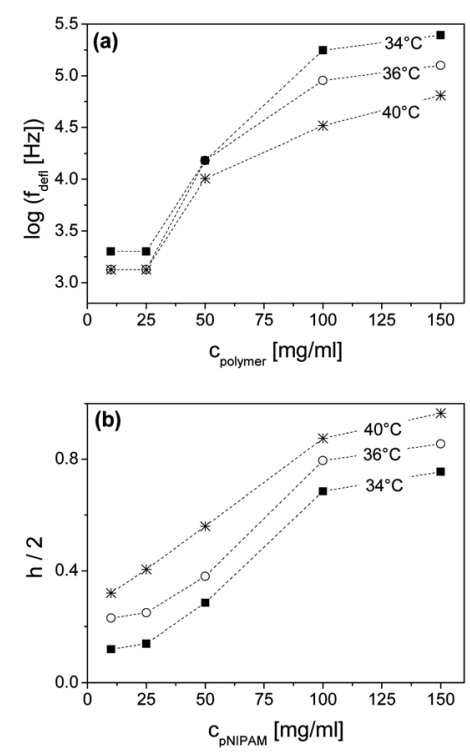

Fig. 10 (a) Frequency position of point of deflection $\log f_{\text {defl }}$ of the MWS polarization in dependence on the polymer concentration for $40^{\circ} \mathrm{C}$ (stars), $36{ }^{\circ} \mathrm{C}$ (open circles) and $34^{\circ} \mathrm{C}$ (filled squares). Dashed lines are guides to the eyes. (b) Step height $h / 2$ of the MWS polarization in dependence on the polymer concentration for $40{ }^{\circ} \mathrm{C}$ (stars), $36{ }^{\circ} \mathrm{C}$ (open circles) and $34{ }^{\circ} \mathrm{C}$ (filled squares). Dashed lines are guides to the eyes.

concentration there is a higher density of soft particles at $T>$ LCST which should lead to more pronounced MWS polarization effects. Second, if the concentration is further increased also larger particles can be formed (due to aggregation) as discussed above. This will increase the MWS polarization effects further. Moreover, it can be seen that the step height increases with increasing temperature at a fixed polymer concentration. This can be related to the kinetics of the phase transition. The transition process from the coiled to the globular state passes different intermediate stages. ${ }^{13}$ For the dielectric measurement the heating/cooling rate was around $0.2 \mathrm{~K} \mathrm{~min}^{-1}$ which means that the system cannot reach the equilibrium state at the LCST since the heating/cooling is too fast (see Fig. 4 and discussion). The increase of the step height with the temperature shows that intermediate states are passed and the fully collapsed (final equilibrium) state is developing with the temperature.

\section{Conclusion}

The phase transition behaviour of aqueous solutions of low molecular linear pNIPAM was investigated using dielectric spectroscopy in a frequency range of $10^{-1} \mathrm{~Hz}$ to $10^{6} \mathrm{~Hz}$ at temperatures between $15^{\circ} \mathrm{C}$ and $50{ }^{\circ} \mathrm{C}$. The dielectric data were discussed in the conductivity representation $\sigma^{*}(f, T)$ since the general approach was to use charges as probes to investigate the internal structure of the pNIPAM solution. It was shown that the phase transition of pNIPAM can be monitored by both the temperature $(T)$ and the frequency $(f)$ dependence of the conductivity spectra. The formation of soft particles at the lower critical solution temperature (LCST) gives rise to a change in the $T$-dependence of the DC conductivity and Maxwell-WagnerSillars (MWS) polarization effects. The latter effect was observed and discussed in the $f$-dependence of the complex conductivity 
for the first time. These effects were mainly related to the change in the charge mobility, its density $\left(\sigma^{*}(T)\right)$ and to charge transport processes including the charge arrangement $\left(\sigma^{*}(f)\right)$ at the LCST.

From the $T$-dependence of the DC conductivity $\sigma_{\mathrm{DC}}^{\prime}$ the following conclusions are drawn:

- The LCST deduced from the change in slope of the $T$ dependence of $\sigma_{\text {DC }}^{\prime}$ shows a hysteresis behaviour (LCST heating $>$ $\mathrm{LCST}_{\text {cooling}}$ ). Besides the fact that any dissolution process has inherent kinetics here, in addition the hysteresis is related to the formation of additional hydrogen bonds in the collapsed state which hinder the refolding/dissolution of the soft particles during cooling.

- The hysteresis ( $\triangle \mathrm{LCST})$ increases with increasing heating/ cooling rate since the chain contraction (heating) as well as the dissolution of the collapsed pNIPAM chains (cooling) cannot follow the temperature change. Furthermore, it was found that different time scales are needed for the formation and the dissolving of hydrogen bonds. For the lowest rate of $0.1 \mathrm{~K} \mathrm{~min}^{-1}$ $\Delta$ LCST vanishes and true equilibrium states can be considered at each temperature.

- At a constant heating/cooling rate $\left(0.2 \mathrm{~K} \mathrm{~min}^{-1}\right) \Delta \mathrm{LCST}$ was investigated in dependence on the polymer concentration. A transition from the dilute to the semidilute and further to a more condensed regime can be observed by abrupt changes in the concentration dependence of $\triangle \mathrm{LCST}$. The estimated critical overlap concentration $C^{*}$ determined corresponds quantitatively to the calculated one.

From the $f$-dependence of the conductivity $\sigma^{\prime}$ the following conclusions were drawn:

- Above the LCST a pronounced $f$-dependence of $\sigma^{\prime}$ was observed for pNIPAM solutions. This is related to a MaxwellWagner-Sillars (MWS) polarization due to the blocking and separation of charges at the formed soft particles (globules).

- The MWS effect characterized by a step-like increase of $\sigma^{\prime}$ with increasing frequency was analysed with respect to the position of its point of deflection and its height in dependence on the polymer concentration. The frequency position of the point of deflection is shifted to higher frequencies with increasing amount of polymer since shorter times (i.e. higher frequency) are needed for charge blocking at interfaces with increasing particle concentration. Moreover, the MWS polarization effect increases with increasing polymer concentration due to a higher density of soft particles and larger particles (aggregation at high polymer concentrations).

To summarize, herein a new approach was reported for the investigation of the phase transition behaviour of aqueous solutions of linear pNIPAM. By taking the charges as probes the thermal collapse of pNIPAM solutions over a wide concentration and temperature range could be investigated. In addition kinetic effects of the phase transition behaviour could be addressed. Dielectric spectroscopy was proven to be a powerful tool to investigate aqueous pNIPAM solution. The obtained results are taken as a starting point for further investigations on different more complex pNIPAM systems. New information can be extracted from dielectric data by analyzing the behaviour of charge carriers. Not only the internal morphology and its influence on the thermal behaviour can be examined also the kinetics of the phase transition can be approached using dielectric spectroscopy.

\section{Acknowledgements}

The authors gratefully acknowledge Dr J. Falkenhagen for GPC and MALDI measurements.

\section{References}

1 M. Das, H. Zhong and E. Kumacheva, Annu. Rev. Mater. Res., 2002, 36, 117.

2 M. Karg and T. Hellweg, Curr. Opin. Colloid Interface Sci., 2009, 14, 438-450.

3 S. Nayak, D. Gan, M. Serpe and L. Lyon, Small, 2005, 1, 416-421.

4 F. Zhang and C. Wang, Colloid Polym. Sci., 2008, 286, 889-895.

5 L. Zhang, E. S. Daniels, V. L. Dimonie and A. J. Klein, J. Appl. Polym. Sci., 2010, 118, 2502-2511.

6 H. Schild, Prog. Polym. Sci., 1992, 17, 163-249.

7 M. Heskins and J. E. Guillet, J. Macromol. Sci., Chem., 1968, 2(8), $1441-1455$.

8 C. Wu and S. Zhou, Phys. Rev. Lett., 1996, 77, 3053-3055.

9 H. Cheng, L. Shen and C. Wu, Macromolecules, 2006, 39, 23252329.

10 C. Cametti, Soft Matter, 2011, 7, 5494-5506.

11 M. Taha, B. S. Gupta, I. Khoiroh and M. J. Lee, Macromolecules, 2011, 44(21), 8575-8589.

12 Y. Kuznetsov, E. Timoshenko and K. Dawson, J. Chem. Phys., 1996, 104, 3338-3347.

13 C. Wu, Polymer, 1998, 39, 4609-4619.

14 P. Zhu and D. Napper, J. Phys. Chem. B, 1997, 101, 3155-3160.

15 J. M. Guenet, Thermoreversible Gelation of Polymers and Biopolymers, Academic Press, San Diego, 1992.

16 K. Zhou, Y. Lu, J. Li, L. Shen, G. Zhang, Z. Xie and C. Wu, Macromolecules, 2008, 41, 8927-8931.

17 C. Wu and X. Wang, Phys. Rev. Lett., 1998, 79, 4092-4094.

18 Y. Maeda, T. Nakamura and I. Ikeda, Macromolecules, 2001, 34, $8246-8251$

19 Y. Katsumoto, T. Tanaka, H. Sato and Y. Ozaki, J. Phys. Chem. A, 2002, 106, 3429-3435.

20 H. Schild and D. Tirrell, J. Phys. Chem., 1990, 94, 4352-4356.

21 Y. Xia, X. Yin, N. Burke and H. Stover, Macromolecules, 2005, 38, 5937-5943.

22 Y. Ding, X. Ye and G. Zhang, Macromolecules, 2005, 38, 904-908.

23 F. Winnik, Macromolecules, 1990, 23, 233-242.

24 Y. Xia, X. Yin, N. Burke and H. Stover, Macromolecules, 2006, 39, 2275-2283.

25 F. M. Winnik, M. F. Ottaviani, S. H. Bossman, W. S. Pan, M. Garcia Garibay and N. J. Turro, J. Phys. Chem., 1993, 97, 12998-13005.

26 J. Spevacek, Macromol. Symp., 2005, 222, 1-13.

27 Broadband Dielectric Spectroscopy, ed. F. Kremer and A. Schönhals, Springer Verlag, Heidelberg, 2003.

28 Y. Ono and T. Shikata, J. Am. Chem. Soc., 2006, 128, 1003010031.

29 Y. Ono and T. Shikata, J. Phys. Chem. B, 2007, 111, 1511-1513.

30 S. Nakano, Y. Sato, R. Kita, N. Shinyashiki, S. Yagihara and S. Sudo, J. Phys. Chem. B, 2012, 116(2), 775-781.

31 F. Gómez-Galván, T. Lara-Ceniceros and H. Mercado-Uribe, Meas. Sci. Technol., 2012, 23(2), 025602.

32 P. Atkins and J. de Paula, Atkins's Physical Chemistry, Oxford University Press, 8th edn, 2006.

33 A. Serghei, M. Tress, J. R. Sangoro and F. Kremer, Phys. Rev. B: Condens. Matter Mater. Phys., 2009, 80, 184301.

34 G. Masci and C. Cametti, J. Phys. Chem. B, 2009, 113, 1142111428 .

35 P. G. de Gennes, Scaling Concepts in Polymer Physics, Cornell University Press, Ithaca, New York, 1979.

36 T. Hu, J. Gao and C. Wu, J. Macromol. Sci., Part B: Phys., 2000, 39(3), 407-414.

37 J. G. Kirkwood and J. Riseman, J. Chem. Phys., 1948, 16, 565.

38 Y. Ding, X. Ye and G. Zhang, J. Phys. Chem. B, 2008, 112, 84968498.

39 T. Hanai, Kolloid-Z., 1960, 171, 23-31.

40 K. Asami, Prog. Polym. Sci., 2002, 27, 1617-1659.

41 M. Böhning, H. Göring, A. Fritz, K. Brzezinka, G. Turky, A. Schönhals and B. Schartel, Macromolecules, 2005, 38, 2764-2774. 